\title{
Research on Innovative Design of Product Packaging
}

\author{
Fan Kang*, Chen Yingyan, Zhou Yangang \\ Wuhan Polytechnic University, Wuhan, China \\ E-mail:Kangfan2004@163.com
}

Keywords: Product packaging design;Cloud computing

\begin{abstract}
The design of product packaging is a bridge of communicating the customers with the enterprise, which also is the key to making mutual benefits. In this paper, it takes the definition of the design of product packaging as the starting point, with the technical support of product packaging design service as well as the interpretation of product packaging design service model based on cloud computing, discussing the designing method of product packaging.
\end{abstract}

\section{Introduction}

It is particularly important for the role of packaging in business competition, which can be regarded as the dominant position in the buyer's market,which even can become a key for the commodity to have successful sales. A reasonable, elegant and distinctive cultural product packaging can meet various demands of customers, which can maximize to stimulate consumer's desire to buy.

\section{The Interpretation of the Design of product Packaging}

In some degree, design is a visual communication program for the intended purpose, in other words, design is not simply the visual language that is attached to the commercials. However, it is the perfect combination between technology and art. The design of product packaging is a part of packaging design, which is one of the important elements of product sales. In all aspects of product packaging design, the designer should be always around product itself to design. The participation of product in product packaging design should be through the visual language to express the perception of product taste, vision, smell and touch, etc..

\section{Supporting Services for the Design of product Packaging Based on Cloud Computing Technology}

Cloud computing is a new kind of computing model based on Internet, which is the further development of the distributed computing, grid computing. It can provide services for enterprises and individuals through the heterogeneous and autonomous services on the Internet with computing resources on-demand; cloud computing resources are dynamically virtualization, which can be provided through the Internet with the calculation of distribution to a large number of distributed computers. American national standards and Technology (NIST) researchers developed a widely used term that is used to describe various aspects of cloud computing. According to the definition of "cloud", it defined three delivery model, which is called S-P-I model, Such as Fig. 1 Schematic Diagram of Cloud Computing Environment. 


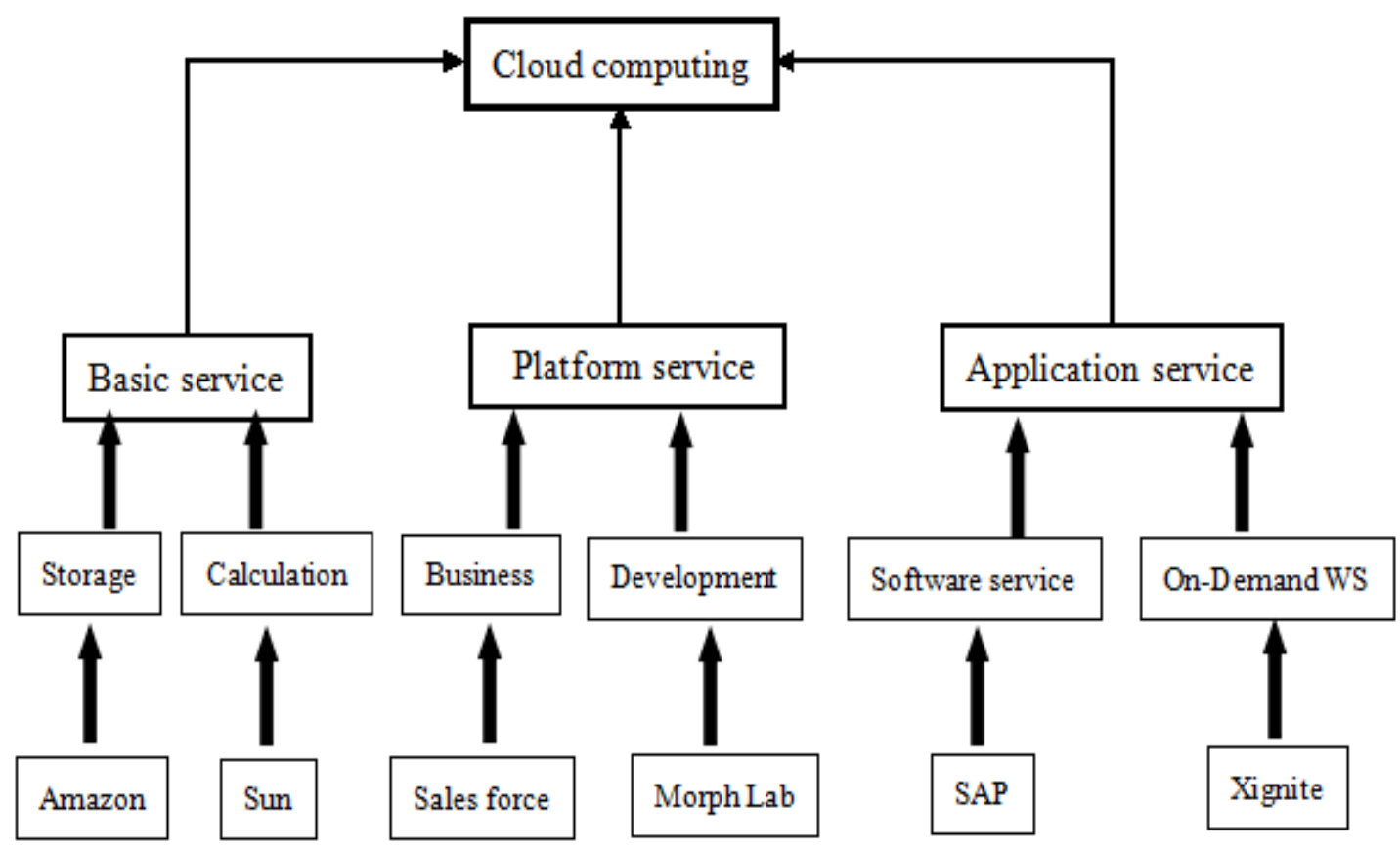

Fig1. Cloud computing model

Cloud computing is used to study the designing services, which is mainly focused on service oriented manufacturing, putting forward the mode of cloud manufacturing at the same time. Cloud manufacturing is a kind of service oriented, with high performance computing and information technology, which is the integration of the existing information, manufacturing technology and cloud computing, networking, semantic Web. It can combine all kinds of manufacturing resources and manufacturing capabilities, as well as service virtualization, which also can be composed of manufacturing resources and manufacturing capacity pool, the intelligent management and operation of a unified centralized, so as to realize the realization of intelligent, win-win, universal and efficient sharing and cooperation, through the network and cloud manufacturing system during the manufacturing process in the whole life cycle. Ultimately, it can provide access on-demand use with safe and reliable, high-quality and cheap intelligence service at any time.

\section{The Model of product Packaging Design Service}

The business mode of product packaging design service can be shown in Fig. 2, the users can buy products from enterprises and pay for what they buy, and the the price of the product is composed of the cost of the enterprise plus profit. According to the analysis of the mode of design service, great changes have taken place during the period of the design process, wile the cost of the design is no longer decided during the designing process at the traditional designing stage, however, it can be divided into smaller units between the designing services, since these designing services units can be in accordance with the parallel implementation, while most of the designing units with high cost of designing services can be completed by the means of outsourcing, therefore, the cost of completing the design by the enterprise itself is the minimal cost for designing the product. Tn a word, the cost of designing product is the total sum of the cost of each designing unit.

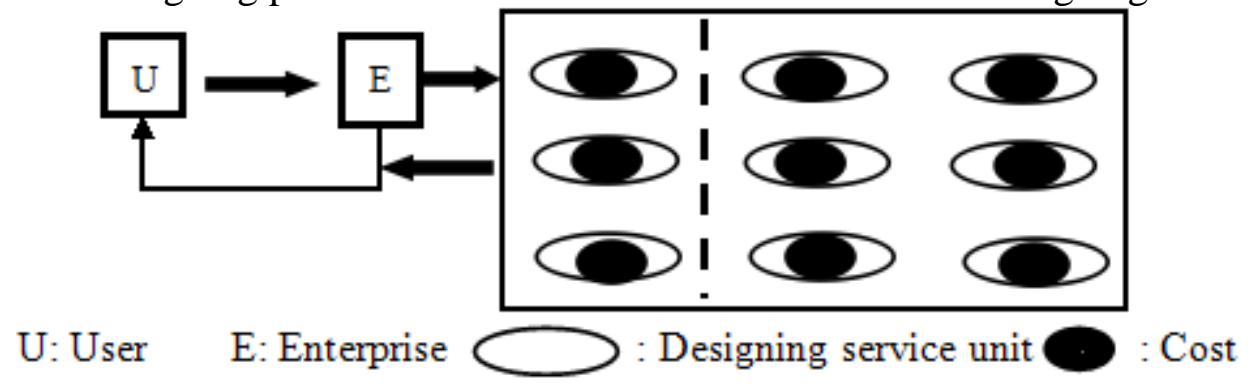

Fig. 2 The design service model 


\section{The Designing Strategy of product Packaging}

\section{Perceptual Performance of Graphics}

Graphics is one of the main elements that can transfer information and show the unique feature of the designing information during the period of designing product packaging. The graphic language has rich meaning, which can be full of strong visual impact, easy memory, obvious feature as well as some other features.Moreover, graphics in packaging is mainly used for showing the packaging performance of visual symbols, text structure form as well as figures, etc.. Therefore, most of the packaging designers pay much attention on the graphic language in packaging design as well as its creative performance. In order to let consumers pay much attention on the product packaging that is designed by the designer at first sight, at the same time, on seeing the visual beauty with the strong impact force, the consumers can form a psychological resonance and have some memory so that they can have the desire to buy the product. In some degree, the graphic language is undoubtedly the most powerful weapon that can show the visual packaging visual beauty with its unique charm.

\section{The Perceptual Performance of Words}

The performance of words is one of the main forms of designing logo for product. The form of words can have better interpretability which can be regarded as the form of the visual language. It can give consumers a strong visual impact and aesthetic feeling, which can convey commodity information well. Therefore, the logo that is mainly used words to show the meaning of the signs can coordinate well with strokes, shade of thickness, so as to reach the overall coordination. Clear and concise feature can be coordinated with the other elements mutually.

\section{The Emotional Expression of Color}

Color can bring people with the visual and psychological shock, which is always incomparable. In some degree, color can provide the products with infinite vitality and vigor, as one of designing elements, color can play a decisive role for the packaging of goods. During the process of designing the product packaging, color can make consumers pay attention to the goods at the first time. Good color design can convey eye-catching and unique visual language, besides, it also can bring people with many concrete or abstract associations, so as to strengthen information cognition and memory of the product.

\section{Perceptual Manifestation of Form}

The form of product packaging design can be composed of two parts, namely, the packaging structure and packaging material, packaging structure can be regarded as the "form language" of product packaging design, which is the only one language that can be reached. Therefore, the quality of packaging structure design and the selection of material can determine whether the consumer can have attention to the goods within a short period of time or not. Through the unique shape and structure as well as the accurate selection of material, designers can control the visual and tactile feeling of the consumers, resulting in imagination and psychological resonance, which can stimulate the potential demand for products and finally give order to buy the products.

\section{Perceptual Performance of Arrangement}

Perceptual expression of the designing arrangement can play a key role in the outlook of product packaging. The style that it showed can be divided into three types: firstly, useful taste, the words which can represent this kind of style including: practicality, without waste, commercial, restrictive feature; secondly, casual taste,the words which can represent this kind of style including: open, relaxed, freely to take part in, freedom, without limitation; lastly, spiritual taste, the words which can represent this kind of style including: suitable for the specific population, appealing, interesting, spiritual, introversion. In this way, it can use the appropriate layout with the various designing elements on the outer surface of the packing, so as to achieve the purpose of conveying information as much as possible and attract more consumers to build brand charm at the same time.As shown in fig3. 


\begin{tabular}{|c|c|c|c|}
\hline & U style & C style & S style \\
\hline 1 & Grid & Free gird & Symmetric \\
\hline 3 & Large & Medium & Small \\
\hline 4 & Square & $\begin{array}{c}\text { Pull the } \\
\text { bottom }\end{array}$ & Bleeding \\
\hline 5 & Text & $\begin{array}{c}\text { Graph \& } \\
\text { Text }\end{array}$ & Graph \\
\hline & Medium & Large & Small \\
\hline
\end{tabular}

Fig. 3 Schematic Chart of the Arrangement of product Packaging Designing Elements

\section{Conclusion}

The design of product packaging is affecting consumer's buying trends, it is a bridge for communicating between consumers and goods. During the process of designing, it should take people as the orientation, it also should meet the needs of people and take it as the original intention of designing, so as to explore how products in product packaging design can have more visual rationality. Moreover, how to be well accepted by the target audience and meet the needs of people's using function and aesthetic function is still very important issue for the design of product packaging.

\section{Acknowledgment}

Introduced talents start-up capital projects in Wuhan polytechnic university: The augmentd reality supported publishing media fusion model in the mobile Internet era. No:20152S05

Humanities and social science project in Hubei province: Origami art innovation applicati -on research in the packaging design No:14G234

\section{Conclusion}

[1]Gisela Kozak and Junlius Wiedemana. 2008. Package Design Now. Italy: TASCHEN GMBH. pp63-64.

[2]Ramsland, T. 1989. Handbook on the procurement of Packaging. Finland:PRODFC. pp55-57.

[3]Edard Denison, Guang Yu Ren. 2001. Thinking Green Packaging Prototypes. UK:Roto Vision S A, pp33-35.

[4]Scott Boylston. 2009. Sustainable Packaging. UK:Laurence King Publishing Ltd.. pp21-22.

[5]Jian-ping Zhu. 2006. Discussing on the Safe Packaging of product. product Science, vol.87, pp566_569. China. 